\title{
A case of congenital giant gallbladder with massive hydrops mimicking celiac cyst
}

\author{
LIANG ZONG ${ }^{*}$, PING CHEN ${ }^{*}$, LEI WANG, CHUNLAN HE, GUANGYAO WANG, \\ JIAN JIANG and HAO WANG
}

Department of Gastrointestinal Surgery, Su Bei People's Hospital, Yangzhou University, Yangzhou, Jiangsu 225001, P.R. China

Received July 7, 2012; Accepted October 3, 2012

DOI: $10.3892 / \mathrm{ol} .2012 .1010$

\begin{abstract}
We present a case of a 55-year-old female who suffered from a mass in the right upper abdomen, which had been present for over six months. Pre-operative blood examinations, including tumor markers, were normal. Initially, the admitting diagnosis was a giant celiac cyst, due to its liquid cystic appearance and large size (approximately $30.0 \times 18.0 \mathrm{~cm}$ ), visible in the hepatic region under ultrasound. Following the discovery of a cystic duct during surgery, the diagnosis was corrected to be a giant gallbladder. As no obstructive matter was observed, the giant gallbladder was considered to be congenital.
\end{abstract}

\section{Introduction}

Large gallbladders are commonly present in biliary diseases, but reports of giant gallbladder are rare (1-4). Previous studies have revealed that a giant gallbladder was always accompanied with a tumor or gallstones. However, extremely large gallbladder with no marked obstructive factors such as biliary tumors and gallstones is considerably rarer. In this case report we present a rare case of a giant gallbladder that contained nothing but extremely large hydrops. This unique case may be useful in exploring the mechanisms involved in the development of giant gallbladders. Written informed consent was obtained from the patient's family.

\section{Case report}

The patient was a 55-year-old female who complained of a mass in the right upper abdomen that had first appeared

Correspondence to: Dr Liang Zong, Gastrointestinal Surgery Department, Su Bei People's Hospital, Yangzhou University, Yangzhou, Jiangsu 225001, P.R. China

E-mail: 250537471@qq.com

*Contributed equally

Key words: large gallbladder over six months previously. Prior to this, the patient had a 5-year-long history of abdominal distension. Until admission, the patient was well; she experienced epigastric discomfort due to compression, but did not experience a fever, vomiting, abdominal ache or jaundice. A physical examination revealed a large lump in the liver area on the right side of the rib cage. This continued down as far as the hip bone, but not past the mid-section, and the patient did not experience any notable tenderness. Routine blood test results, including hepatic function and renal function, were found to be normal. An abdominal ultrasound scan revealed a giant, liquid, dark area measuring $30.0 \times 18.0 \mathrm{~cm}$, visible in the hepatic region (Fig. 1). No abnormalities were revealed in the laboratory data, including the tumor markers that are listed in Table I.

A laparotomy was performed, during which an incision was made in the middle line, below the umbilicus. Initially, it was not possible to determine the point at which the sac originated, due to its large size of approximately $30.0 \times 31.0 \times 18.0 \mathrm{~cm}$. The sac constituted almost half of the total epigastric region. Additionally, it was not possible to discern the layers of peritoneum and sac, owing to its very firm adhesion. As the uncertainty concerning the sac was so great, it was decided that the sac be emptied with a large trocar; approximately $3,800 \mathrm{ml}$ of fluid was drained, leaving at least $200 \mathrm{ml}$ in the bottom of the cyst. The diagnosis was corrected to be a giant gallbladder when a $4 \mathrm{~mm}$ slender cystic duct connecting to an $8 \mathrm{~mm}$ choledochus was discovered during the operation. As no obstructive matter, such as a tumor or gallstones were observed, this particular giant gallbladder was considered to be congenital. The pathological section revealed that columnar epithelium was present in the lining of the gallbladder wall along with inflammatory cell infiltration (Fig. 2A and B). Following the operation, there were no complications. The patient was treated and discharged after 10 days.

\section{Discussion}

A normal gallbladder is typically no more than $7.5-10 \mathrm{~cm}$ in length. However, it can present a considerably large size under the rare condition of genoconstitution. However, most gallbladders of extremely large size are correlated 
Table I. Laboratory findings on admission.

\begin{tabular}{|c|c|c|}
\hline Blood markers & Index & Normal range \\
\hline WBC (x109/1) & 10.1 & $4.0-10.0$ \\
\hline $\mathrm{RBC}\left(\mathrm{x} 10^{12} / \mathrm{l}\right)$ & 3.7 & $3.5-5.0$ \\
\hline BUN (mmol/l) & 5.6 & $3.2-6.0$ \\
\hline Hemoglobin (g/l) & 113 & $110-150$ \\
\hline Hematocrit (vol\%) & 40 & $37-48$ \\
\hline Platelets $\left(\mathrm{x} 10^{9} / 1\right)$ & 210 & $100-3001$ \\
\hline Total protein $(\mathrm{g} / \mathrm{l})$ & 67 & $60-80$ \\
\hline Albumin/globulin ratio & 1.2 & $1.1-2.5$ \\
\hline Total bilirubin $(\mu \mathrm{mol} / \mathrm{l})$ & 11.2 & $5.7-23.5$ \\
\hline Direct bilirubin $(\mu \mathrm{mol} / \mathrm{l})$ & 2.3 & $1.7-7.8$ \\
\hline SGOT (U/l) & 37 & $0-50$ \\
\hline SGPT (U/l) & 42 & $0-50$ \\
\hline Total cholesterol (mmol/l) & 1.30 & $1.29-1.55$ \\
\hline Serum amylase (U/l) & 61 & $25-125$ \\
\hline Lactic dehydrogenase (U/l) & 147.0 & $135.0-215.0$ \\
\hline Alkaline phosphatase (U/l) & 76 & $25-150$ \\
\hline CA19-9 (KU/1) & 5.47 & $<35.00$ \\
\hline CA242 (KU/l) & 1.31 & $<20.00$ \\
\hline CA125 (KU/l) & 2.27 & $<35.00$ \\
\hline CA15-3 (KU/1) & 2.28 & $<35.001$ \\
\hline NSE (ng/ml) & $<1.0$ & $<13.00$ \\
\hline CEA (ng/ml) & 1.21 & $<5.00$ \\
\hline Ferritin (ng/ml) & 27.13 & $<219.00$ \\
\hline Bta-HCG (MIU/ml) & $<0.021$ & $<3.00$ \\
\hline $\operatorname{AFP}(n g / m l)$ & 0.88 & $<20.00$ \\
\hline Free-PSA (ng/ml) & $<0.22$ & $<1.00$ \\
\hline PSA (ng/ml) & $<0.04$ & $<5.00$ \\
\hline HGH (ng/ml) & 2.15 & $<7.50$ \\
\hline
\end{tabular}

with pathological states, particularly in obstructive biliary diseases. The original study by Courvoisier et al stated that a growing, large gallbladder was more frequently caused by a biliary obstructive tumor such as a pancreatic malignancy, rather than gallstones, which may attribute to intraluminal hypertension over a shorter period of time (5-7). According to Courvoisier et al, a gallstone does not result in an enlarged gallbladder, as it is formed over an extended period of time, resulting in a shrunken, fibrotic and scarred gallbladder that

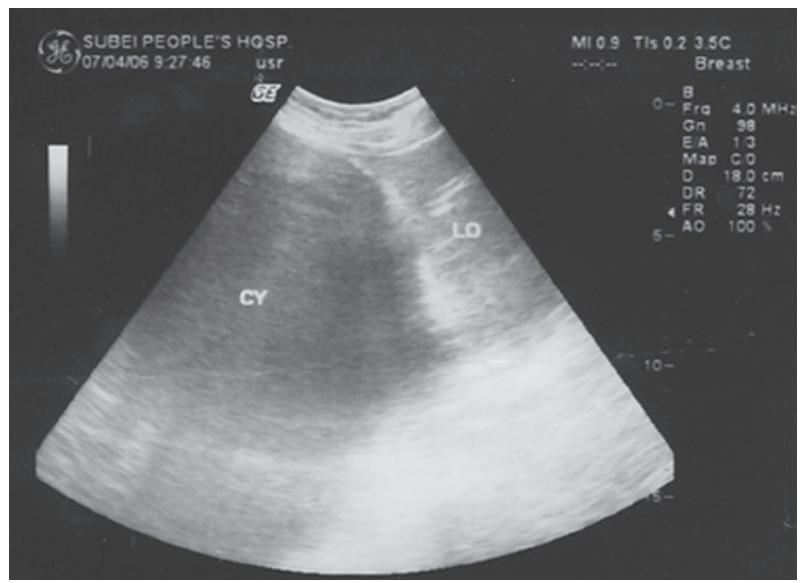

Figure 1. Abdominal ultrasound scan, demonstrating an extremely large, liquid, dark area, approximately $18.0 \mathrm{~cm}$ in depth, visible in the hepatic region.

\section{A}

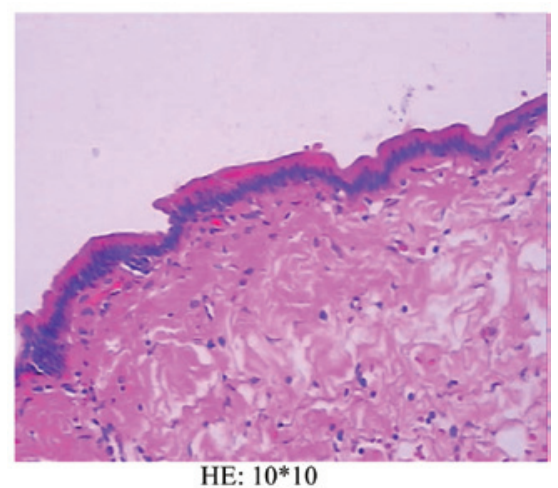

B

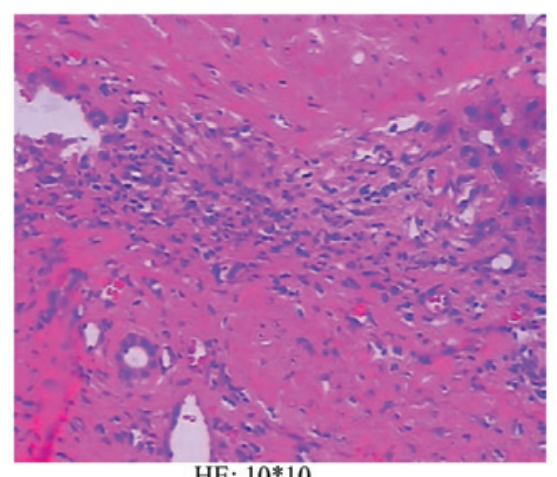

Figure 2. Pathological section with hematoxylin and eosin (HE) staining, demonstrating columnar epithelium present in the lining of the gallbladder wall with inflammatory cell infiltration.

Table II. Overview of the reported cases of giant gallbladder.

\begin{tabular}{lccccc}
\hline Authors (Ref.) & Gender & Age (years) & Size (cm) & Obstruction & Post-operative diagnosis \\
\hline Grosberg (1) & Female & 95 & $14 \times 5.5$ & Stone & Acute gangrenous cholecystitis, cholelithiasis \\
Maeda et al (2) & Female & 36 & $18 \times 4$ & No & Chronic cholecystitis, cholelithiasis \\
Hsu et al (3) & Female & 87 & $16.4 \times 13.6 \times 7.8$ & No & Acute cholecystitis, gall bladder adenocarcinoma \\
Panaro et al (4) & NA & 17 & $43 \times 21 \times 20$ & No & Byler's disease \\
\hline
\end{tabular}

NA, not available. 
does not allow extended enlargement. However, an exception to Courvoisier's law supports the theory that the stones may be responsible for a growing, large gallbladder. This theory suggests that the stones may dislodge and acutely block the duct distally to the hepatic/cystic duct junction, contributing to the action of check valves. In a review of published work (1), such cases of a giant gallbladder combined with gallstones can be further explained by this theory of etiology.

The case we present here was unique, owing to the unusually large size of the gallbladder, being amongst the largest found in a review of the literature (Table II). Moreover, no obstructive factors, such as a tumor or stones, were present. However, the biliary duct was fluent, which enabled bile to freely enter the intestinal tract. As neither marked biliary inflammation nor biliary obstruction was observed, this particular giant gallbladder was considered to be congenital. With the progression of the disease, the giant gallbladder became increasingly filled with bile, which lead to chronic inflammation that subsequently damaged the contractile function of the gallbladder and contributed to further growth of the congenital large gallbladder.

\section{References}

1. Grosberg SJ: Giant gallbladder. Am J Dig Dis 7: 1039-1040, 1962.

2. Maeda Y, Setoguchi T, Yoshida T, et al: A giant gallbladder. Gastroenterol Jpn 14: 621-624, 1979.

3. Hsu KF, Yeh CL, Shih ML, et al: Giant gallbladder: adenocarcinoma complicated with empyema. J Trauma 70: 261, 2011.

4. Panaro F, Chastaing L and Navarro F: Education and imaging. Hepatobiliary and pancreatic: giant gallbladder associated with Byler's disease. J Gastroenterol Hepatol 27: 620, 2012.

5. Hagege A: Case of retention jaundice; importance of CourvoisierTerrier law. Tunis Med 45: 439, 1957 (In French).

6. Watts GT: Courvoisier's law. Lancet 2: 1293-1294, 1985.

7. Verghese A and Berk SL: Courvoisier's law. Lancet 1: 99, 1986. 